\title{
INEQUALITIES AND BOUNDS FOR A CERTAIN BIVARIATE ELLIPTIC MEAN II
}

\author{
EDWARD NEUMAN
}

Abstract. Further investigations of the bivariate elliptic mean introduced in [?] are presented. In particular new bounds for the mean under discussion are obtained. Also, the Wilker and Huygens-type inequalities as well as the Landen transformation are established. Results presented in this work are complimentary to those derived recently in [?] and [?].

Mathematics subject classification (2010): 26E30, 26D05, 33C05.

Keywords and phrases: Bivariate means; Schwab-Borchardt mean; inequalities; Gauss hypergeometric function; Wilker and Huygens-type inequalities; Landen's transformation.

\section{REFERENCES}

[1] J.M. Borwein, P.B. Borwein, Pi and the AGM-A Study in Analytic Number Theory and Computational Complexity, Wiley, New York, 1987.

[2] B.C. Carlson, Algorithms involving arithmetic and geometric means, Amer. Math. Monthly, 78 (1971) 496-505.

[3] B.C. Carlson, Special Functions of Applied Mathematics, Academic Press, New York, 1977.

[4] S.-B. Chen, Z.-Y. He, Y.-M. Chu, Y.-Q. Song, X.-J. Tao, Note on certain inequalities for Neuman means, J. Inequal. Appl. 2014, 2014:370, 10 pages.

[5] Z.-J. Guo, Y.-M. Chu, Y.-Q. Song, X.-J. Tao, Sharp bounds for Neuman means by harmonic, arithmetic, and contra-harmonic means, Abstr. Appl. Anal. Volume 2014, Article ID914242, 8 pages.

[6] Z.-J. Guo, Y. Zhang, Y.-M. Chu, Y.-Q. Song, Sharp bounds for Neuman means in terms of geometric, arithmetic and quadratic means, arXiv: 1405, 4384v1, May 2014.

[7] Y.-M. Li, B.-Y. Long, Y.-M. Chu, Sharp bounds for the Neuman-Sándor mean in terms of generalized logarithmic mean, J. Math. Inequal., 4 (2012) 567-577.

[8] E. Neuman, Inequalities for the Schwab-Borchardt mean and their applications, J. Math. Inequal., 5 (2011) 601-609.

[9] E. Neuman, A note on a certain bivariate mean, J. Math. Inequal., 6 (2012) 637-643.

[10] E. Neuman, Inequalities for weighted sums of powers and their applications, Math. Inequal. Appl., 15 (2012) 995-1005.

[11] E. Neuman, Sharp inequalities involving Neuman-Sándor and logarithmic means, J. Math. Inequal., 7 (2013) 413-419.

[12] E. Neuman, Inequalities involving certain bivariate means, J. Inequal. Spec. Functions, 4 (2013) 12 20.

[13] E. Neuman, A one-parameter family of bivariate mean, J. Math. Inequal., 7 (2013) 399-412.

[14] E. Neuman, On some means derived from the Schwab-Borchardt mean, J. Math. Inequal. 8 (2014) 171-183.

[15] E. Neuman, On some means derived from the Schwab-Borchardt mean II, J. Math. Inequal., 8 (2014) 361-370.

[16] E. Neuman, On a new bivariate mean, Aequat. Math., 88 (2014) 277-289.

[17] E. Neuman, Inequalities involving generalized trigonometric and hyperbolic functions, J. Math. Inequal., 8 (2014) 725-736.

[18] E. Neuman, Optimal bounds for certain bivariate means, Issues of Analysis., 7(21) (2014) 35-43.

[19] E. Neuman, On a new bivariate mean II, Aequat. Math., 89 (2015) 1031-1040. 
[20] E. Neuman, Inequalities and bounds for a certain bivariate elliptic mean, Math. Inequal. Appl., 19 (2016) $1375-1385$.

[21] E. Neuman On two bivariate means, J. Math. Inequal., 11 (2017) 345-354.

[22] E. Neuman, J. Sándor, On the Schwab-Borchardt mean, Math. Pannon., 14 (2003) 253-266.

[23] E. Neuman, J. Sándor, On the Schwab-Borchardt mean II, Math. Pannon., 17 (2006) 49-59.

[24] K. C. Richards, Sharp power mean bounds for the Gaussian hypergeometric function, J. Math. Math. Anal. Appl., 308 (2005) 303 - 313.

[25] W.-M. Qian, Y.-M. Chu, Refinements and bounds for Neuman means in terms of arithmetic and contraharmonic means, J. Math. Inequal., 9 (2015) 873-881.

[26] W.-M. Qian, Z.-H. Shao, Y.-M. Chu, Sharp inequalities involving Neuman means of the second kind and other bivariate means, J. Math. Inequal., 9 (2015) 531-540.

[27] H.-J. Seiffert, Problem 887, Nieuw. Arch. Wisk., 11 (1993) 176.

[28] H.-J. Seiffert, Aufgabe 16, Würzel, 29 (1995) 87.

[29] Y. Zhang, Y.-M. Chu, Y.-L. Jiang, Sharp geometric mean bounds for Neuman mean, Abstr. Appl. Anal., Volume 2014, Article ID 949815, 6 pages.

[30] T.-H. Zhao, Y.-M. Chu, B.-Y. Liu, Optimal bounds for Neuman-Sándor mean in terms of arithmetic and contra-harmonic means, Abstr. Appl. Anal., Volume 2012, Article ID 302635, 9 pages. 